\title{
Determining The Types Of Tiles Based On Building Location And Building Elevation Using Fuzzy Weighted Product (WP) Methods
}

\author{
Sayyidah $^{1}$, Nurul Huda Maksum ${ }^{2}$, Sarmiatul Hasanah $^{3}$, Tony Yulianto ${ }^{4}$ \\ \{sayydhaams@gmail.com ${ }^{1}$, nurulhuda@gmail.com ${ }^{2}$, sarmiatulhasanah@gmail.com ${ }^{3}$ \} \\ 1,2,3,4 Mathematics Department, Mathemathics and Science Faculty, Islamic University of Madura, \\ Pamekasan, Madura, Indonesia
}

\begin{abstract}
Tile is one of the basic ingredients in making roofs of houses or other buildings that are widely used by the people of Indonesia. The types of tile have several types so that the determination is determined based on the location of the building and the height of the building that is different, so that from this case the fuzzy weighted product method is applied to determine the type of tile. Data taken from 40 tile buyers was carried out by direct observation of tile makers, where there were types of clay tiles including plentong tile, manthili tile, garuda tile, frog tile. The criteria used are the location of the building, building height and temperature. The results of the highest ranking of vector $\mathrm{V}$ are found in the second alternative, namely manthili tile with a building location of $170.5 \mathrm{~m} /$ above sea level, building height of $7.712 \mathrm{~cm}$ and temperature / weather of $27.99^{\circ} \mathrm{C}$.
\end{abstract}

Keywords: Tile, fuzzy, weighted product, ranking.

\section{Introduction}

Tile is one of the basic ingredients in making roofs or other buildings that are widely used by Indonesian people. The demand for tile continues to increase along with the number of people who want to build and rehabilitate their homes. For this reason, making tile can be a promising business opportunity [1].

Tile is the main part of a building as a roof covering the house. The main function of the tile is to resist the heat of the sun and the splash of rain. Types of tile vary, there are concrete roof tiles, clay tiles, ceramic tiles, zinc tile, and wood tile (shingle), but in this study we will study the type of clay tile because of the superiority of clay tiles in addition to cheap materials that can withstand all weather and lighter than concrete tile. While the weakness of this tile can break because of falling objects or receiving a large pressure load exceeding its capacity. The quality of the tile is very determined from the material and combustion temperature, because it will determine the water absorbency and the power of tile pressure, therefore efforts to improve the quality and quality of tile from clay continue to be pursued along with the exit of various roofs for example: asbestos, zinc, tile print from large industries and others [2].

In this study to be able to produce a good tile can be seen in the location of the building and the height of the building. So to determine the type of tile on the location of the building affects the type of tile because the location of the building is higher in the air temperature than the lowlands and the rainfall that is taking place is also quite high so it requires a type of tile that is strong, thick, and has sufficient combustion temperature. Whereas in the lowlands the air temperature is lower and the rainfall that takes place is quite low so it requires a type of tile 
that is strong, thin, and has sufficient combustion temperature. While based on the height of the building to determine the type of tile it is influential because if the building is higher (floored) the tile is thicker. Conversely, if the height of the building is low (not floored), the tile will be thinner for that, there needs to be a method that can minimize roof loads and costs. Many methods can determine the type of tile based on the location of the building and the height of the building, one method that can be used is the Fuzzy Weighted Product (WP) method

Fuzzy logic is one component of the formation of soft computing. Basic Fuzzy Logic is Fuzzy set theory. Fuzzy set theory is the role of membership degree as a determinant of the existence of elements in a set is very important. Membership value or degree of membership is the main characteristic of reasoning with fuzzy logic. Fuzzy is an appropriate way for the input space into the expected output space. Weighted Product (WP) is a mathematical model that is used to do alternative ranking by looking for weighted multiplication to connect the attribute rating where the rating of each attribute must be raised first with attribute weights. concerned [3].

Based on previous research conducted by Syaukani (2012) entitled "Modeling of Clinical Decision Support Systems with Fuzzy Weighted Product (WP) Method" the results of his research found new ways to diagnose pneumonia in adults. Also carried out by Buhari (2012) about Determining Ibn Malik's Alphabet Book Memorization Using the Weighted Product Method (WP) "the results of his research found a new way to memorize by listening chosen as the best way to memorize the book Alfiyah Ibn Malik"

Based on this background the researcher was interested in conducting a study entitled "Determination of Tile Types Based on Building Location and Building Height Using the Fuzzy Weighted Product (WP) Method." Hopefully it can help users know the type of tile that is appropriate and appropriate to the building location and height

\section{Definition of Fuzzy Logic}

Fuzzy logic is an appropriate way to map an input space into an output space. The fuzzy set is based on the idea of extending the range of characteristic functions so that the function will include real numbers at intervals [0,1]. [4].

\section{Weighted Product}

Weighted Product Method (WP) is a method used to do alternative ranking by looking for weighted multiplication to connect attribute rating where rating of each attribute must be raised first with the weight of the attribute in question. Stages in the WP method [5].

Make improvements to the weight with the equation as follows:

$$
w_{j}=\frac{w_{j}}{\sum w_{j}}
$$

Based on equation (1) calculating the value of vector $S$ is given as follows:

$$
S_{i}=\prod_{j=1}^{n} X_{i j}^{w j}, i=1,2, \ldots . m
$$

with:

$i$ : Alternative

$m$ : Many Alternatives

$n$ : Many Criteria

$S$ : Alternative diagonal preference as vector $\mathrm{S}$

$W$ : Weight criteria / subcriteria 
with: $\sum W_{j}=1 . \mathrm{W}_{\mathrm{j}}$ is a positive value for the profit attribute and is negative for the cost attribute. Then from equation (2) the process of ranking the value of vector $\mathrm{V}$ is carried out as follows:

with:

$$
V_{i}=\frac{s_{i}}{\sum_{j=1}^{n} s_{i}}, i=1,2, \ldots . m
$$

$V$ : The relative preference of each alternative is analogous to vector $\mathrm{V}$

\section{Fuzzy Weighted Product}

Decision making is generally done by choosing the best alternative from a number of alternatives. If the selection of the best alternative is done by considering certain criteria, then the process is called multi attribute decision making (MADM). One method that is widely used in MADM is a weighted product.

The use of fuzzy logic in the weighted product method is due to the presence of data in the form of linguistics (qualitative) in an alternative. For example, tile type A has a "GOOD" resistance to building location and building height. Whereas type B tiles have resistance to building locations and the height of buildings that are "LESS GOOD". So the concept of fuzzy logic is very appropriate if combined with the weighted product method in solving the problem of decision making [6].

Using the following equation to determine each expert's preference:

$$
P_{i j}^{k}=\frac{\left(u_{i}^{k}\right)^{2}}{\left(u_{i}^{k}\right)^{2}+\left(u_{j}^{k}\right)^{2}} ; 1 \leq i \neq j \leq m
$$

with:

$u_{i}^{k}=$ preference value given by experts to alternatives.

$P_{i j}^{k}=$ value of each expert.

Aggregation Preferences

If Fuzzy linguistic quantifiers are used in linguistic form, "most", then we can obtain the weight vector for preference using the following equation:

with :

$$
w_{i}=Q\left(\frac{i}{n}\right)-Q\left(\frac{i-1}{n}\right) i=1, \ldots, n
$$

$w_{i}=$ value of preference aggregation

$Q=$ criteria value (expert)

$i=$ the highest value in the set of expert preferences $\left(P_{1}, P_{2}, P_{3}\right)$

Next, the elements of each row in the matrix $\left(P_{1}, P_{2}, P_{3}\right)$ of equation (4) are sorted down so that the matrix matrix $P^{c}$ is obtained below:

with:

$$
P^{c}=\phi \operatorname{most}\left(P_{1}, P_{2}, P_{3}\right)
$$

$P^{c}=$ aggregation matrix

$\Phi$ most $=$ preference weight vector

The $P^{c}$ matrix of equation (6) is not yet a normal matrix. For this reason, it is necessary to normalize the $P^{c} \mathrm{c}$ matrix to get the $P^{c^{\prime}}$ matrix with the following formula:

$$
P_{i j}^{c \prime}=\frac{P_{i j}^{c}}{P_{i j}^{c}+P_{j i}^{c}}
$$


with:

$P^{c}=$ the value of the preference aggregation matrix based on rows and columns

$P^{c^{\prime}}=$ preference aggregation matrix

$i=$ value from the matrix line of preference

$j=$ value from the matrix column of preference

Then from equation (7) weights each of the following features:

$$
w_{i}=\frac{1}{n} \sum_{j} a_{i j}^{i}
$$

with:

$w_{i}=$ weight of goal $i$ of the weight vector

After collecting data, the algorithm is carried out as follows:

1. Determine the preference (criteria) of the expert $\left(P_{i j}^{k}\right)$ using equation (4)

2. Determine preference aggregation weights using equation (5)

3. Create a preference aggregation matrix $\left(P^{c}\right)$ using equation (6)

4. Normalize the preference aggregation matrix $\left(P^{c^{\prime}}\right)$ using equation (7)

5. Determine the feature weight $\left(W_{i}\right)$ using equation (8)

6. Repair weight $\left(W_{j}\right)$ using equation (1)

7. Calculate weighted preferences $(S)$ using equation (2)

8. Calculate preference value $(\mathrm{V})$ using equation (3)

9. Do ranking

\section{Results and Discussion}

The first process that must be done is to determine the expert preferences (criteari) $\left(P_{i j}^{k}\right)$. Where each expert is assumed to give his preference to use linguistic forms. Preferred is given as follows:

Plentong tile expert $=\{$ Low, Low, High $\}$

Manhtili tile expert $=\{$ High, Very High, High $\}$

Garuda tile expert $=\{$ Very High, Very High, Low $\}$

Toad tile expert $=\{$ Low, Very Low, High $\}$.

The following is a linguistic form which is represented as a triangular fuzzy number in the building location categorized as follows:

$$
\begin{aligned}
& \text { Very High }=(0.66667 ; 0.83333 ; 1) \\
& \text { Height }=(0.5 ; 0.66667 ; 0.83333) \\
& \text { Moderate }=(0.33333 ; 0.5 ; 0.66667) \\
& \text { Low }=(0.16667 ; 0.33333 ; 0.5) \\
& \text { Very Low }=(0 ; 0,16667 ; 0,33333)
\end{aligned}
$$

The linguistic form represented as a triangular fuzzy number at the height of the building is categorized as follows:

$$
\begin{aligned}
& \text { Very High }=(0.85185 ; 0.92593 ; 1) \\
& \text { Height }=(0.77778 ; 0.85185 ; 0.92593) \\
& \text { Medium }=(0.7037 ; 0.77778 ; 0.85185) \\
& \text { Low }=(0.62963 ; 0.7037 ; 0.77778) \\
& \text { Very Low }=(0.55556 ; 0.62963 ; 0.7037)
\end{aligned}
$$

The linguistic form represented as triangular fuzzy numbers at temperature / weather is categorized as follows: 
Very High $=(0,96045 ; 0,98023 ; 1)$

Height $=(0.96068 ; 0.96045 ; 0.98023)$

Medium $=(0,9209 ; 0,94068 ; 0,96045)$

Low $=(0.90113 ; 0.9209 ; 0.94068)$

Very Low $=(0.88136 ; 0.90113 ; 0.9209)$

Using equation (4) then to determine each expert preference as follows:

$$
P_{i j}^{k}=\frac{\left(u_{i}^{k}\right)^{2}}{\left(u_{i}^{k}\right)^{2}+\left(u_{j}^{k}\right)^{2}} ; 1 \leq i \neq j \leq m
$$

For example for plentong tile experts give a value of preference: $\{$ Low, Low, High $\}$.

$$
\begin{aligned}
& P_{11}^{1}=\frac{(0,33333)^{2}}{(0,33333)^{2}+(0,33333)^{2}}=\frac{0,11111}{0,11111+0,11111}=\frac{0,11111}{0,22222}=0,5 \\
& P_{12}^{1}=\frac{(0,33333)^{2}}{(0,33)^{2}+(0,7037)^{2}}=\frac{0,11111}{0,11111+0,49519}=\frac{0,11111}{0,6063}=0,1833 \\
& P_{13}^{1}=\frac{(0,33333)^{2^{2}}}{(0,33333)^{2}+(0,96045)^{2}}=\frac{0,11111}{0,11111+0,92246}=\frac{0,11111}{1,03357}=0,1075
\end{aligned}
$$

So that in the same way the preferences of each expert matrix were obtained, namely $P^{1}$ as the plentong tile expert preference matrix, $P^{2}$ as the manthili tile expert preference matrix, , $P^{3}$ as the Garuda tile tile preference matrix, $P^{4}$ as the expert preference matrix frog tile, which is the second process to find the preference matrix of each expert, following the results of each preference matrix:

$$
\begin{aligned}
P^{1} & =\left[\begin{array}{lll}
0,5000 & 0,1833 & 0,1075 \\
0,8167 & 0,5000 & 0,3493 \\
0,8925 & 0,6507 & 0,5000
\end{array}\right] \\
P^{2} & =\left[\begin{array}{lll}
0,5000 & 0,3414 & 0,3251 \\
0,6586 & 0,5000 & 0,4817 \\
0,6749 & 0,5183 & 0,5000
\end{array}\right] \\
P^{3} & =\left[\begin{array}{lll}
0,5000 & 0,4475 & 0,4502 \\
0,5525 & 0,5000 & 0,5027 \\
0,5498 & 0,4973 & 0,5000
\end{array}\right] \\
P^{4} & =\left[\begin{array}{lll}
0,5000 & 0,2189 & 0,1075 \\
0,7811 & 0,5000 & 0,3006 \\
0,8925 & 0,6994 & 0,5000
\end{array}\right]
\end{aligned}
$$

Furthermore, the third process is Aggregation Preference where by using equation (5) when Fuzzy linguistic quantifiers are used in linguistic form, "most", we can obtain weight vectors for preferences as follows:

$$
\begin{aligned}
& W_{1}=Q\left(\frac{1}{4}\right)-Q\left(\frac{0}{4}\right)=\sqrt{\frac{1}{4}}-\sqrt{\frac{0}{4}}=0,5 \\
& W_{2}=Q\left(\frac{2}{4}\right)-Q\left(\frac{1}{4}\right)=\sqrt{\frac{2}{4}}-\sqrt{\frac{1}{4}}=0,207 \\
& W_{3}=Q\left(\frac{3}{4}\right)-Q\left(\frac{2}{4}\right)=\sqrt{\frac{3}{4}}-\sqrt{\frac{2}{4}}=0,1589 \\
& W_{4}=Q\left(\frac{4}{4}\right)-Q\left(\frac{3}{4}\right)=\sqrt{\frac{4}{4}}-\sqrt{\frac{3}{4}}=0,1340
\end{aligned}
$$

With equation (6) the fourth process is to find the $P^{c}$ matrix, where to find the $P^{c}$ matrix, the value of the preference weight vector is multiplied by the matrix $\left(P_{1}, P_{2}, P_{3}, P_{4}\right)$ of the equation ( 9 ) to (12) so that the $P^{c}$ matrix is obtained as follows: 


$$
\begin{gathered}
=(0,5) P_{1}+(0,207)+(0,1589) P_{3}+(0,1340) P_{4} \\
P^{C}=\left[\begin{array}{ccc}
0,5 & 0,2628 & 0,2070 \\
0,7372 & 0,5 & 0,3946 \\
0,7930 & 0,6054 & 0,5
\end{array}\right]
\end{gathered}
$$

The next process is the fifth process, namely normalization of the preference aggregation matrix $\left(P^{c^{\prime}}\right)$, where the $P^{c}$ matrix of equation (13) is not yet a normal matrix. For this reason, it is necessary to normalize the $P^{c}$ matrix to get the matrix $P^{c^{\prime}}$ with the formula using equation (7) below:

$$
\begin{aligned}
& P_{11}^{c \prime}=\frac{0,5}{0,5+0,5}=\frac{0,5}{1}=0,5 \\
& P_{12}^{c \prime}=\frac{0,2628}{0,2628+0,7372}=\frac{0,2628}{1}=0,2628 \\
& P_{13}^{c \prime}=\frac{0,2070}{0,2070+0,7930}=\frac{0,2070}{1}=0,2070
\end{aligned}
$$

Then from this process, the matrix values $P^{c^{\prime}}$ can be generated as follows:

$$
P^{c^{\prime}}=\left[\begin{array}{ccc}
0,5 & 0,2628 & 0,2070 \\
0,7372 & 0,5 & 0,3946 \\
0,7930 & 0,6054 & 0,5
\end{array}\right]
$$

Then from equation (14), the sixth process is obtained by the weight of each feature so that it can be calculated based on equation (8) as follows:

$$
\begin{aligned}
& w_{1}=\frac{1}{3}(0,5+0,2628+0,2070) \\
& =\frac{1}{3}(0,9698)=0,3233 \\
& w_{2}=\frac{1}{3}(0,7372+0,5+0,3946) \\
& =\frac{1}{3}(1,6318)=0,5439 \\
& w_{3}=\frac{1}{3}(0,7930+0,6054+0,5) \\
& =\frac{1}{3}(1,8984)=0,6328
\end{aligned}
$$

After obtaining feature $\mathrm{w}$ weights, the seventh process using the weighted product method in equation (1) is carried out to repair the following weights:

$$
\begin{aligned}
& w_{j}=\frac{w_{j}}{\sum w_{j}} \\
& w_{1}=\frac{0,3233}{0,3233+0,5439+0,6328}=0,2155 \\
& w_{2}=\frac{0,5439}{0,3233+0,5439+0,6328}=0,3626 \\
& w_{3}=\frac{0,6328}{0,3233+0,5439+0,6328}=0,4219
\end{aligned}
$$

Based on the data obtained the value of $\mathrm{X}$ with the results of normalization as follows:

$$
\text { Values } X=\left[\begin{array}{lll}
0,2064 & 0,2226 & 0,2509 \\
0,2931 & 0,2700 & 0,2502 \\
0,2779 & 0,2600 & 0,2487 \\
0,2226 & 0,2474 & 0,2503
\end{array}\right]
$$


Then after knowing the value of $\mathrm{x}$ in equation (15), the eighth process is to calculate the value of vector $\mathrm{S}$ based on equation (2) as follows:

$$
\begin{aligned}
S_{i} & =\prod_{j=1}^{n} X_{i j}^{w j} \\
S_{1} & =\left(0,2064^{0,2155}\right)\left(0,2226^{0,3626}\right)\left(0,2509^{0,4219}\right) \\
& =0,23033 \\
S_{2} & =\left(0,2931^{0,2155}\right)\left(0,2700^{0,3626}\right)\left(0,2502^{0,4219}\right. \\
& =0,2661 \\
S_{3} & =\left(0,2779^{0,2155}\right)\left(0,2600^{0,3626}\right)\left(0,2487^{0,4219}\right. \\
& =0,25886 \\
S_{4} & =\left(0,2226^{0,2155}\right)\left(0,2474^{0,3626}\right)\left(0,2503^{0,4219}\right) \\
& =0,24303
\end{aligned}
$$

Then after finding the value of vector $S$, the ninth process is done, namely ranking the value of vector $\mathrm{V}$ based on equation (3) as follows:

$$
\begin{aligned}
& V_{i}=\frac{S_{i}}{\sum_{j=1}^{n} S_{i}} \\
& V_{1}=\frac{0,23033}{0,23033+0,2661+0,25886+0,24303}=0,23072 \\
& V_{2}=\frac{0,2661}{0,23033+0,2661+0,25886+0,24303}=0,26655 \\
& V_{3}=\frac{0,25886}{0,23033+0,2661+0,25886+0,24303}=0,25930 \\
& V_{4}=\frac{0,24303}{0,23033+0,2661+0,25886+0,24303}=0,24344
\end{aligned}
$$

After calculating the relative preference of each alternative (vector $\mathrm{V}$ ) it can be ranked. The results of the ranking of plentong tile, tile manthili, tile Garuda, tile toad can be seen in Table 1 as follows:

Table 1 Ranking

\begin{tabular}{cccccc}
\hline \multirow{2}{*}{ Alternative } & \multicolumn{3}{c}{ Criteria } & \multirow{2}{*}{ Vector V } & \multirow{2}{*}{ Rank } \\
\cline { 2 - 4 } & Building Location & Building Height & Temperature / Weather & & \\
\hline Plentong & 120,1 & 6,358 & 28,069 & 0,23072 & 4 \\
Manthili & 170,5 & 7,712 & 27,99 & 0,26655 & 1 \\
Garuda & 161,7 & 7,426 & 27,822 & 0,25930 & 2 \\
Kodok & 129,5 & 7,068 & 28,009 & 0,24344 & 3 \\
\hline
\end{tabular}

\section{Conclusions}

Based on the simulation results using fuzzy weighted product, it can be concluded that the highest $V$ value is found in the second alternative, ie manthili tile with a building location of $170.5 \mathrm{~m}$ above sea level, building height $7.712 \mathrm{~cm}$ and temperature / weather $27.99 \mathrm{oC}$.

\section{Acknowledgments}

The author would like to thank:

1. Ristek Dikti which has provided funding assistance related to PKM PE.

2. Dean, Chair of the UIM MIPA Study Program and Supervisor who have PKM PE.

3. The Palengaan tile craftsmen have collaborated in this research. 


\section{References}

[1] Afiyak, M., 2015. Making Tile. Saturday January, p. 4.

[2] Prihartono, E., 2010. Analysis of Tile Burning Heat Transfer. In: Thesis. Surakarta: Muhammadiyah University Surakarta, p. 2.

[3] Syaukani, M., 2012. Modeling of Clinical Decision Support Systems with Fuzzy Weighted Product Method. article, p. 42.

[4] Yulianto, T. \& Amalia, R., 2017. Decision Making of Potential Salt Positions on the Coast of Madura Using Fuzzy K-Nearest Neighbor (FKNN). In: Research. Pamekasan: Madura Islamic University, p. 10.

[5] Buhari, 2012. Determination of the Best Memorandum of Events in the Book Alfiyah Ibnu Malik Using the Weighted Product Method. In: thesis. Pamekasan: Madura Islamic University, p. 10.

[6] Syaukani, M. \& Kusnanto, H., 2012. Modeling of Group Decision Support Systems with Fuzzy Weighted Product Methods for Diagnosis of Pneumonia. Journal of Technology, Volume 5 number 1, p. 20. 